\title{
Binary Tree Approach for Data Hiding Based on Histogram Modification
}

\author{
P.D.Ratna Raju \\ Associate Prof \\ Priyadarshini Institute of Tech \& Science
}

\author{
B.A.David \\ Associate Prof \\ Krest Technologies
}

\author{
K.Prasada Rao \\ Professor \\ Chandrakala P.G. College
}

\begin{abstract}
This paper presents a new approach for data hiding scheme for the secure digital data transmission. Here we present the modification to histogram by considering the differences of the pixels which can be used for increasing data hiding capacity. We also show-off how different images affect the performance of this proposed method.
\end{abstract}

Key words: watermarking, authentication, Histogram, Binary tree

\section{INTRODUCTION}

In these days where we can find a rapid growth in the field on information technology, for human communication on the internet we are in the need of providing high security for our digital data. Hiding the data in imperceptible manner may be one of the solutions for this. How ever there are few images which are not tolerable and very sensitive for embedding distortions such as military images, medical images and artwork preservation.

Many techniques have been proposed earlier for data hiding using G-LSB data hiding techniques [1] which uses a variant arithmetic compression algorithm to encode the message and hide the resulting interval number in the host image. L.M Cheng proposed an enhancement for the conventional LSB (least significant bit) by considering an optimal pixel adjustment process for hiding data.

Diljith M.thodi and Jefferey J used Tian's [6] algorithm based difference expansion (DE) for implementing reversible watermarking. It also included histogram shifting method for the reversible data hiding [3].

In this present paper we extend the histogram modification using pixel differences to increase the capacity of data hiding .we also use binary tree structure approach for multiple of peak points with histogram shifting for overflow and underflow.

\section{PROPOSED METHOD}

In this we propose using histogram peak and zero points.
Let $\mathrm{P}$ be the value at peak point and $\mathrm{Z}$ be value at zero point then if once a pixel value with $P$ is encountered the pixel value is increased by 1,otherwise no modification are required. Extraction of the data is exactly the replicate of data hiding process. Number of message bits that can be embedded can be equal to the number of pixels associated with the peak point [7].Here we present histogram modification technique (only pixel value) by considering the difference of the adjacent pixels. Correlations between the neighboring pixels are very strong and the distribution pixel difference has a prominent maximum, which is expected to be very close zero. This is shown in the figure 1 below

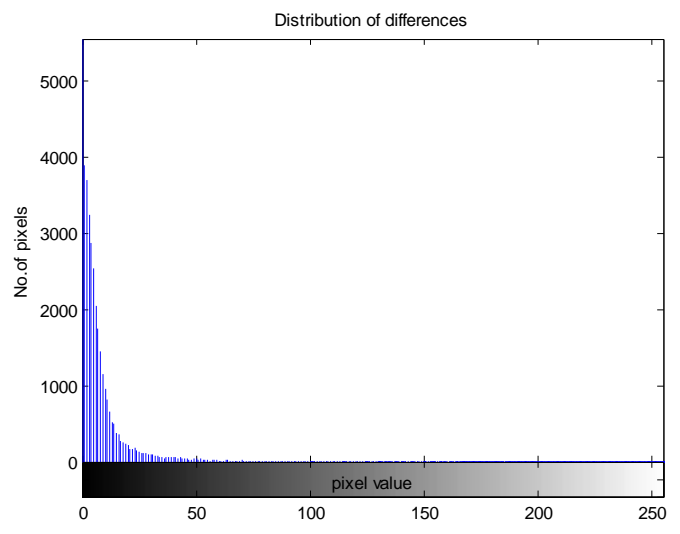

Fig 1: Distribution of pixel differences

By observing the above figure we can embed the done in pixel differences. we also use tree structure approach to solve the problem of communicating multiple pairs of peak points to recipients.

Let us explain the proposal in detail. Consider an N-pixel 8-bit gray level image (I) with the pixel values $x_{i}$, where $i$ denote the number of pixel. i.e.; $0 \leq \mathrm{i} \leq \mathrm{N}-1$ and $\mathrm{x}_{\mathrm{i}} \in \mathrm{I} \mathrm{x}_{\mathrm{i}} \in\left[\begin{array}{ll}0 & 255\end{array}\right]$

The following are the steps to embed the data

1) Scan the image $I$ in the inverse order and calculate the pixel differences 


$$
d i=\left\{\begin{array}{lr}
x_{i} & i=0 \\
x i-1-x i_{v} & \text { otherwise }
\end{array}\right.
$$

2) Determine the peak point $P$ from the differences and based on the shift the pixels by 1 unit

$\mathrm{y}_{\mathrm{i}}= \begin{cases}x i & \text { if } i=0 \text { or } d i<P \\ x i+1 & \text { if } d i>P \text { and } x i \geq x i-1 \\ x i-1 & \text { if } d i>P \text { and } x i<x i-1\end{cases}$

Where $\mathrm{y}_{\mathrm{i}}$ is the watermarked image

3) If $\mathrm{d}_{\mathrm{i}}=\mathrm{P}$ then modify $x_{\mathrm{i}}$ according to the message bit.

$$
\mathrm{yi}= \begin{cases}x i+b & \text { if } \text { di }=P \text { and } x i \geq x i-1 \\ x i-b & \text { if } d i=P \text { and } x i<x i-1\end{cases}
$$

Where $\mathrm{b}$ is the message bit to be embed At the receiver end the recipient extracts the message from watermarked image by performing the same reverse scanning

The following are the steps to extract he data

1) The message can be extracted by

$$
\mathrm{b}= \begin{cases}0 & \text { if }\|y i-x i-1\|=P \\ 1 & \text { if }\|y-x i-1\|=P+1\end{cases}
$$

Where $x_{\mathrm{i}-1}$ denotes the restored value of yi and can be restored by

$$
x i=\left\{\begin{array}{lr}
y i+1 & \text { if }|y i-x i-1|>P \text { and } y i<x i-1 \\
y i-1 & \text { if }|y i-x i-1|>P \text { and } y i>x i-1 \\
y i & \text { otherwise }
\end{array}\right.
$$

An exact copy of the original image is obtained .In the above mentioned process includes only one peak point. For higher data capacity we extend this for binary tree approach.

A example for the above mentioned approach is as follows

Let the cover image be

\begin{tabular}{|l|l|l|l|}
\hline 154 & 155 & 156 & 157 \\
\hline 155 & 156 & 154 & 157 \\
\hline 159 & 160 & 161 & 158 \\
\hline 157 & 158 & 159 & 160 \\
\hline
\end{tabular}

$x_{i}=\left[\begin{array}{lllll}154 & 155 & 156 & 157155156154157159160161158157158\end{array}\right.$ 159 160];

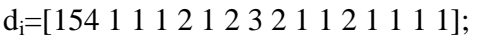

Let the peak point be $\mathrm{P}=2$

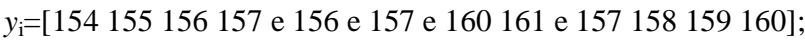

where ' $\mathrm{e}$ ' is the pixel to be calculated from equations (2) \& (3). Then the resultant

let the message be 0110

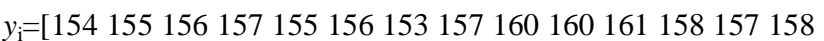
$159160]$

Extraction using equation (4) \& (5) restored

$x_{i}=\left[\begin{array}{llll}154 & 155156157155156154157159160161158157158\end{array}\right.$ 159 160];

Compare restored $x_{\mathrm{i}}$ and $y_{\mathrm{i}}$ at peak points then 0110 is the extracted message

\section{Binary Tree approach}

Let us assume that there are $\mathrm{K}$ level of the binary tree then the number of peak points used to embed messages are $2^{\mathrm{K}}$. Once the pixel difference $d_{\mathrm{i}}$ satisfies $d_{\mathrm{i}}<2^{\mathrm{K}}$ then if the message bit to be embedded is " 0 " the left child of the node $d_{\mathrm{i}}$ is visited other wise the right one see figure 2 . Higher payloads require the higher level. However, all the recipient needs to share with the sender is the tree level $\mathrm{K}$, because we propose an auxiliary binary tree that pre-determines multiple peak points used to embed messages.

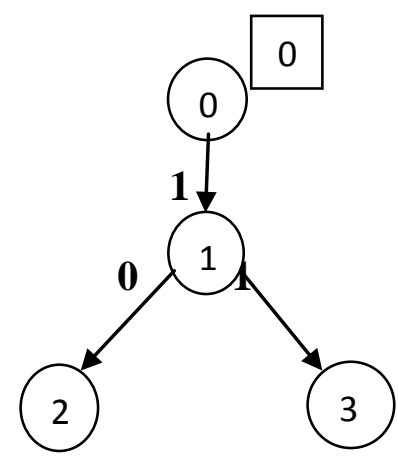

Fig 2: Auxiliary binary 2 level tree 
The following are the steps for embedding

Consider an N-pixel 8-bit gray level image (I) with the pixel values $x_{i}$, where $i$ denote the number of pixel. i.e.; $0 \leq \mathrm{i} \leq \mathrm{N}-1$ and $\mathrm{x}_{\mathrm{i}} \in \mathrm{I} \mathrm{x}_{\mathrm{i}} €\left[\begin{array}{ll}0 & 255\end{array}\right]$

1) Determine the level $K$ of binary tree

2) Shift the histogram from both sides by $2^{\mathrm{K}}$ units. This is done to control the over flow and under flow of histograms

3) Scan the entire image in inverse order and calculate the pixel differences between $\mathrm{x}_{\mathrm{i}-1}$ and $\mathrm{x}_{\mathrm{i}}$

4) If $d_{i} \geq 2^{K}$ shift by $2^{K}$ units

$\mathrm{y}_{\mathrm{i}}= \begin{cases}x i & \text { if } i=0 \\ x i+\mathbb{L} & \text { if } d i>\mathbb{L} \text { and } x i \geq x i-1 \\ x i-\mathbb{L} & \text { if } d i>\text { PLand } x i<x i-1\end{cases}$

where $\mathrm{L}=2^{\mathrm{K}}$.

5) If $\mathrm{d}_{\mathrm{i}}<2^{\mathrm{K}}$ modify $x_{\mathrm{i}}$

$\mathrm{y}_{\mathrm{i}}= \begin{cases}x i+(d i+b) & \text { if } x i \geq x i-1 \\ x i-(d i+b) & \text { if } x i<x i-1\end{cases}$

Where $b$ is the message to be embedded $b\{0.1\}$.

The following are the steps for extraction

Let $\mathrm{K}$ be the proposed level for the binary tree.

For an N-pixel 8-bit gray level image (y) with the pixel values $\mathrm{y}_{\mathrm{i}}$, where $\mathrm{i}$ denote the number of pixel i.e.; $0 \leq \mathrm{i} \leq \mathrm{N}-1$ and $\mathrm{y}_{\mathrm{i}} € \mathrm{I}$ $\mathrm{x}_{\mathrm{i}} \in\left[\begin{array}{ll}0 & 255]\end{array}\right.$

1) Scan the entire image in inverse order and if $\mid y_{i}, x_{i-}$ ${ }_{1} \mid<2^{\mathrm{K}+1}$ then

$\mathrm{b}= \begin{cases}0 & \text { if } \| y-x i-1 \text { is even } \\ 1 & \text { if }\|y-x i-1\| \text { is odd }\end{cases}$

Where $x_{\mathrm{i}-1}$ denotes the restored value of yi and can be restored $\mathrm{b} x \mathrm{xi}=$

$$
\begin{cases}y i+\frac{y i-x i-1}{2} \mid & \text { if } \mid y i-x i-1 \|<T \text { and } y i<x i-1 \\ y i-\frac{y i-x i-1}{2} & \text { if }|y i-x i-1|<T \text { and } y i>x i-1 \\ y i+T & \text { if }|y i-x i-1| \geq T \text { and } y i<x i-1 \\ y i-T & \text { if }|y i-x i-1| \geq T \text { and } y i>x i-1 \\ y i & \text { othemwise }\end{cases}
$$

Repeat the above step for the entire message to embed.

\section{Experimental results}

All the experiments were conducted with the $256 \times 256$ image like "Lena", "baboon", "hat". For the Lena image
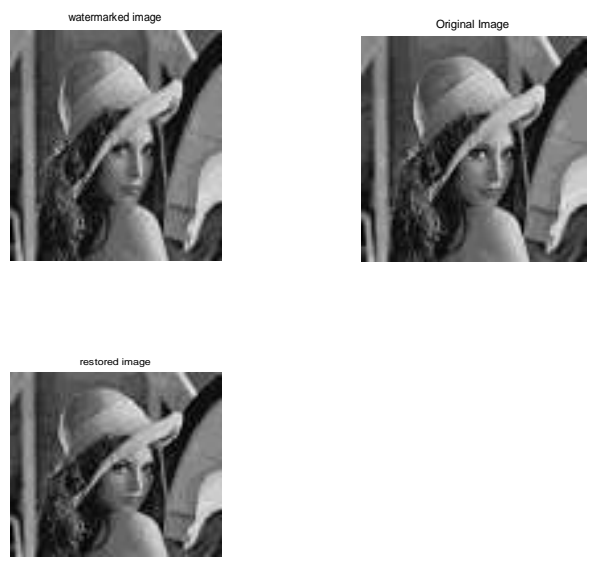

Fig 3: a) Original image b) watermarked c) Restored

\begin{tabular}{|l|l|l|}
\hline Image & PSNR & MSE \\
\hline Lena & 44.28 & 2.422 \\
\hline Baboon & 44.19 & 2.472 \\
\hline Hat & 44.34 & 2.392 \\
\hline
\end{tabular}

All these results are obtained with $\mathrm{bpp}=0.1250$

\section{Capacity}

Thus, the real capacity Cap, that is referred to as pure payload is Cap $=\mathrm{Np}-|\mathrm{O}|$, where $\mathrm{Np}$ is the number of pixels that are associated with peak points and $|O|$ is the length of the overhead information.

\begin{tabular}{|l|l|l|l|}
\hline Image & $\mathrm{Np}$ & Cap & Overhead \\
\hline Lena & 22390 & 22367 & 23 \\
\hline Baboon & 25530 & 25480 & 50 \\
\hline Hat & 22560 & 22524 & 36 \\
\hline
\end{tabular}

\section{Conclusion}

In this paper we have not considered any comparison methods. In future this will be presented. As far as the method is concerned it's very easy to understand and implement. The main limitation of these histogram modification techniques is that we have to provide a side communication channel for pairs of peak points and minimum points. We also mentioned binary tree that predetermines the multiple peak points used to embed messages; thus, the only information the sender and recipient must share is 
the tree level $\mathrm{K}$. This made us to embed large amount of data for hiding, there by increasing its capacity

\section{REFERENCES}

[1] Lossless Generalized LSB data embedding by M.U celik IEEE-2005

[2] Hiding data in images by simple LSB substitution by Chi kwong chan ,L.M cheng

[3] Expansion Embedding techniques for reversible watermarking by Diljith M.thodi and Jefferey J IEEE 2007

[4] Lossless Data embedding for all image formats by Jessica Fedrich ,M.Goljan in SPIE security watermarking multimedia contents IV san Jose CA vol 4675

[5] Data Hiding fundamentals in image and video Part I by Min Mu,Bede Liu IEEE 2003

[6] Reversible Data embedding using a difference expansion J.Tian IEEE-2003 vol 13 No 8

[7] Reversible watermarking using modified difference expansion method by Md.K.Yakub ,ahmed Al Jaber vol 4 No.3 Internal journal of computing and information sciences.
[8] Lossless data embedding with file size preservation by Jessica Fedrich, M.Goljan,Qing chen

[9] Wavelet based reversible watermarking for authentication by J.Tian

[10] Reversible image authentication based on watermarking by sang kwang lee ,Young Ho suh and Yo sung ho IEEE-06-2006

\section{Authors}

a) P.D.Ratna Raju: $\mathrm{He}$ has pursued M.phil from Madurai Kamraj university and presently doing his research on image processing. He is also serving as the head of computer science department at Priyadarshini Engineering college tenali. His research interests includes, security algorithm, image authentication systems

b) B.A.David: $\mathrm{He}$ has pursued M-tech from JNTU (Hyderabad).Presently working on the development of data security algorithm. His research areas include image processing, signal processing speech processing and security algorithms development

c) K.Prasada Rao: $\mathrm{He}$ is currently working as a Principal for Chandrakala PG college Tenali. He has vast teaching experience and providing extensible research guidance for the scholars. 